\title{
Harmonic Oscillator SUSY Partners and Evolution Loops ${ }^{\star}$
}

\author{
David J. FERNÁNDEZ \\ Departamento de Física, Cinvestav, A.P. 14-740, 07000 México D.F., México \\ E-mail: david@fis.cinvestav.mx \\ URL: http://usuarios.fis.cinvestav.mx/david/ \\ Received May 28, 2012, in final form July 04, 2012; Published online July 11, 2012 \\ http://dx.doi.org/10.3842/SIGMA.2012.041
}

\begin{abstract}
Supersymmetric quantum mechanics is a powerful tool for generating exactly solvable potentials departing from a given initial one. If applied to the harmonic oscillator, a family of Hamiltonians ruled by polynomial Heisenberg algebras is obtained. In this paper it will be shown that the SUSY partner Hamiltonians of the harmonic oscillator can produce evolution loops. The corresponding geometric phases will be as well studied.
\end{abstract}

Key words: supersymmetric quantum mechanics; quantum harmonic oscillator; polynomial Heisenberg algebra; geometric phase

2010 Mathematics Subject Classification: 81Q60; 81Q05; 81Q70

\section{Introduction}

In the last decades there has been a growing interest in studying evolution loops (EL), which are circular dynamical processes such that the evolution operator of the system becomes the identity at a certain time $[30,33,37,39,40,44,45,48,50,51,53,54]$. They represent a natural generalization to what happens for the harmonic oscillator. Their importance rests on the fact that the EL are quite sensitive to external perturbations, so they are a good starting point to implement the dynamical manipulation for approximating an arbitrary unitary operator [48] (see also [29]).

On the other hand, the polynomial Heisenberg algebras (PHA) are deformations of the Heisenberg-Weyl algebra in which the commutators of the Hamiltonian $H$ with the annihilation $L^{-}$and creation $L^{+}$operators are standard but the commutator between the last two turns out to be a polynomial in $H[1,3,6,9,13,14,17,18,27,35,38,46,47,49,60]$. In order to characterize the spectrum of $H, \operatorname{Sp}(H)$, one needs to determine the eigenstates of $H$ which are extremal (annihilated by $L^{-}$) and have physical interpretation: thus, $\operatorname{Sp}(H)$ is composed of several independent ladders (either of finite or infinite lengths) departing from those extremal states (for alternative deformations of the Heisenberg-Weyl algebra see e.g. [23, 25, 42, 57]).

In addition, nowadays it is widely accepted that supersymmetric quantum mechanics (SUSY QM) is the simplest technique for generating new Hamiltonians $H_{k}$ departing from a given initial one $H_{0}$ (for recent books and review articles see $[5,10,12,21,26,31,34,41,55,59]$ ). After applying the method, it turns out that $\operatorname{Sp}\left(H_{k}\right)$ differs little from $\operatorname{Sp}\left(H_{0}\right)$ (the differences rely in a finite number of levels). Moreover, the algebraic structure of $H_{k}$ can be obtained straightforwardly of the corresponding algebra of $H_{0}$. In particular, the SUSY partners of the harmonic oscillator Hamiltonian turn out to be ruled by polynomial Heisenberg algebras [35, 49]: this is the simplest way for realizing in a non-trivial way such non-linear algebras.

\footnotetext{
${ }^{\star}$ This paper is a contribution to the Special Issue "Superintegrability, Exact Solvability, and Special Functions".
} The full collection is available at http://www.emis.de/journals/SIGMA/SESSF2012.html 


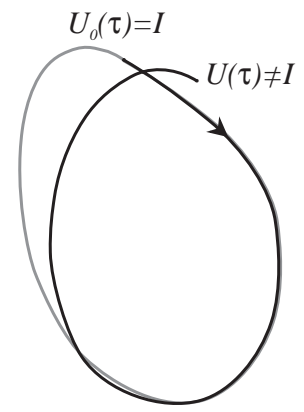

Figure 1. A system which performs an evolution loop at $t=\tau$ when perturbed, in general, will deviate from this cyclic dynamical process.

In this paper I would like to explore in detail the possibility that the SUSY partners Hamiltonians of the harmonic oscillator can have EL, as it happens for the initial system. If the answer becomes positive, it is natural to evaluate then the geometric phase, associated to an arbitrary initial state which is cyclic $[2,4,15,16,19,30,39,40,44,45,56,58]$. It is worth to notice that some partial results along this way have been derived previously [30]. However, they were found just for the Abraham-Moses family of potentials isospectral to the harmonic oscillator [49]. Here, we are going to generalize these results for a SUSY transformation of order $k \geq 1$ so that $H_{0}$ and $H_{k}$ are not necessarily isospectral [35].

The paper has been organized as follows. In the next section we shall introduce briefly the evolution loops, with a discussion about the geometric phases which naturally will arise for systems satisfying such operator identity. In Section 3 the polynomial Heisenberg algebras will be presented in general, while in Section 4 the same shall be done for supersymmetric quantum mechanics. The SUSY partners of the harmonic oscillator will be derived at Section 5, including the analysis of their connections with polynomial Heisenberg algebras, evolution loops and associated geometric phases. Our conclusions shall be presented at Section 6 .

\section{Evolution loops}

At operator level, the dynamics of a quantum system is determined by its evolution operator $U(t)$, which satisfies:

$$
\frac{d U(t)}{d t}=-i H(t) U(t), \quad U(0)=I,
$$

where $H(t)$ is the system Hamiltonian, $I$ is the identity operator, $U(t)$ is unitary. As it was pointed out previously, we are interested in studying system having evolution loops, i.e., circular dynamical processes such that $U(t)$ becomes the identity (up to a phase factor) at a certain time,

$$
U(\tau)=e^{i \varphi} I,
$$

with $\tau>0$ being the loop period, $\varphi \in \mathbb{R}[30,48,51]$. The simplest system with an evolution loop of period $\tau=2 \pi$ is the harmonic oscillator since

$$
\begin{aligned}
& U(t)=\sum_{n=0}^{\infty} e^{-i H t}\left|\psi_{n}\right\rangle\left\langle\psi_{n}\left|=e^{-i t / 2} \sum_{n=0}^{\infty} e^{-i n t}\right| \psi_{n}\right\rangle\left\langle\psi_{n}\right| \Rightarrow \\
& U(\tau)=e^{-i \pi} \sum_{n=0}^{\infty}\left|\psi_{n}\right\rangle\left\langle\psi_{n}\right|=-I,
\end{aligned}
$$




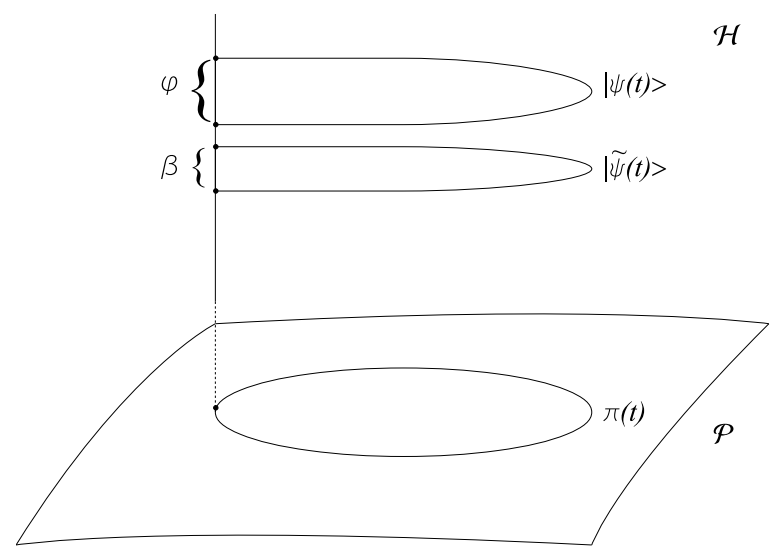

Figure 2. Representation of the cyclic state $|\psi(t)\rangle \in \mathcal{H}$ induced by Schrödinger equation, its closed shadow $\pi(t) \in \mathcal{P}$ and the corresponding horizontal lifting $|\widetilde{\psi}(t)\rangle \in \mathcal{H}$. The holonomy of this lifting is the geometric phase factor $e^{i \beta}$.

where we have employed that $H\left|\psi_{n}\right\rangle=E_{n}\left|\psi_{n}\right\rangle$ with $E_{n}=n+1 / 2, n=0,1, \ldots$ (we are using natural units such that $\hbar=m=\omega=1$ ). The EL are important since they can be used as a starting point to implement the dynamical manipulation in order to approximate any unitary operator $[48,50,51]$. In fact, there is a prescription for implementing this kind of manipulation, which was introduced some years ago [48]: first of all the system has to be placed in an EL, i.e., $U_{0}(\tau) \equiv I$; by perturbing then the EL, the small deviations of this dynamical process such that $U(\tau) \neq I$ will eventually approximate an arbitrary unitary operator (see an illustration in Fig. 1).

It is worth to notice that the EL have been mainly studied for systems ruled by timedependent Hamiltonians, either in one or several dimensions [37, 45, 48, 50, 51, 53, 54] or for purely spin systems $[39,40,44]$. However, there are several works where the evolution loops are produced by time-independent Hamiltonians [30, 33]. In particular, an interesting physical system of such a type consists of a charged particle inside an ideal Penning trap [33].

Since it is an operator relationship (compare with [28]), the requirement of equation (1) is quite strong: it implies that for a system having an EL any $|\psi\rangle \in \mathcal{H}$, taken as an initial condition, becomes cyclic with period $\tau$ :

$$
|\psi(\tau)\rangle=U(\tau)|\psi\rangle=e^{i \varphi}|\psi\rangle .
$$

Thus, it is natural to ask if the global phase $\varphi$ has a geometric component. As it was noticed by Aharonov and Anandan [2], it turns out that a geometric phase $\beta$ can be associated to any cyclic evolution $|\psi(t)\rangle$ satisfying equation (2):

$$
\beta=\varphi+\int_{0}^{\tau}\langle\psi(t)|H(t)| \psi(t)\rangle d t
$$

In particular, if the system is ruled by a time-independent Hamiltonian $H(t)=H$ the previous expression becomes simpler [30]:

$$
\beta=\varphi+\tau\langle\psi|H| \psi\rangle \text {. }
$$

Let us note that $\beta$ describes a global "curvature effect" arising on the space of physical states of the system, which is the projective space $\mathcal{P}$ formed by the rays or the density operators $|\psi\rangle\langle\psi|$ instead of the Hilbert space $\mathcal{H}[2,16,30,39,40]$. Due to this curvature, the horizontal lifting (parallel transport) of the closed trajectory $\pi(t)=|\psi(t)\rangle\langle\psi(t)| \in \mathcal{P}$ leads to a trajectory $|\widetilde{\psi}(t)\rangle$ which is, in general, open on $\mathcal{H}$. The holonomy of this lifting is the Aharonov-Anandan geometric phase factor $e^{i \beta}$ (see Fig. 2). 


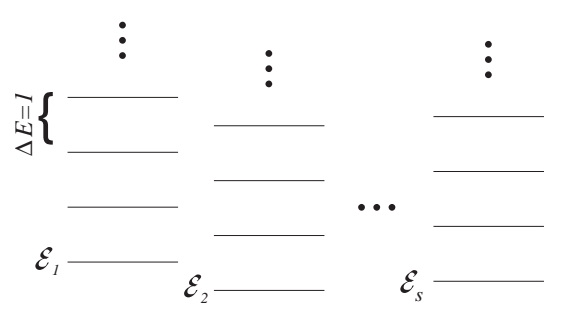

(a)

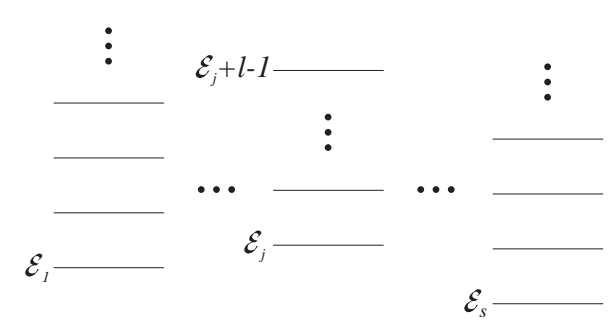

(b)

Figure 3. Spectrum of the Hamiltonians ruled by polynomial Heisenberg algebras: (a) it is composed of $s$ infinite ladders starting from $\mathcal{E}_{j}, j=1, \ldots, s$; (b) the $j$-th ladder becomes finite since equation (7) is satisfied.

\section{Polynomial Heisenberg algebras}

The polynomial Heisenberg algebras are deformations of the Heisenberg-Weyl algebra of kind $[17,35]$ :

$$
\begin{aligned}
& {\left[H, L^{ \pm}\right]= \pm L^{ \pm},} \\
& {\left[L^{-}, L^{+}\right] \equiv Q_{m+1}(H+1)-Q_{m+1}(H)=P_{m}(H),}
\end{aligned}
$$

where

$$
Q_{m+1}(H)=L^{+} L^{-}=\prod_{i=1}^{m+1}\left(H-\mathcal{E}_{i}\right)
$$

is a $(m+1)$-th order polynomial in $H$ which implies that $P_{m}(H)$ is a polynomial of order $m$-th in $H$. A simple way of realizing the algebra of equations (4)-(6) is to suppose that $H$ has the standard Schrödinger form,

$$
H=-\frac{1}{2} \frac{d^{2}}{d x^{2}}+V(x)
$$

while $L^{ \pm}$are $(m+1)$-th order differential operators.

Note that $\operatorname{Sp}(H)$ depends on the number of eigenstates of $H$ belonging to the kernel of $L^{-}$ which have physical meaning. If $s$ of them are physically acceptable and satisfy

$$
L^{-} \psi_{\mathcal{E}_{i}}=0, \quad H \psi_{\mathcal{E}_{i}}=\mathcal{E}_{i} \psi_{\mathcal{E}_{i}}, \quad i=1, \ldots, s,
$$

thus, $\operatorname{Sp}(H)$ turns out to be composed of $s$ independent infinite ladders, each one of them starting from $\psi_{\mathcal{E}_{i}}, i=1, \ldots, s$ (see Fig. 3a).

On the other hand, for the $j$-th ladder which starts from $\psi_{\mathcal{E}_{j}}$ it could happen that

$$
\left(L^{+}\right)^{l-1} \psi_{\mathcal{E}_{j}} \neq 0, \quad\left(L^{+}\right)^{l} \psi_{\mathcal{E}_{j}}=0
$$

for some integer $l$. In this case it turns out that [35]

$$
\mathcal{E}_{n}=\mathcal{E}_{j}+l, \quad n \in\{s+1, \ldots, k\}
$$

which means that the $j$-th ladder starts from the eigenvalue $\mathcal{E}_{j}$ and ends at $\mathcal{E}_{j}+l-1$, i.e., it is a finite ladder of length $l$, with $l$ steps (see Fig. 3b). 


\section{Supersymmetric quantum mechanics}

Let us consider now the following chain of intertwining relationships:

$$
\begin{aligned}
& H_{i} A_{i}^{+}=A_{i}^{+} H_{i-1}, \\
& A_{i}^{ \pm}=\frac{1}{\sqrt{2}}\left[\mp \frac{d}{d x}+\alpha_{i}\left(x, \epsilon_{i}\right)\right], \quad i=1, \ldots, k,
\end{aligned}
$$

where

$$
H_{i}=-\frac{1}{2} \frac{d^{2}}{d x^{2}}+V_{i}(x), \quad i=0, \ldots, k .
$$

By plugging the expressions (9), (10) into equation (8), it turns out that the following must be satisfied:

$$
\begin{aligned}
& \alpha_{i}^{\prime}\left(x, \epsilon_{i}\right)+\alpha_{i}^{2}\left(x, \epsilon_{i}\right)=2\left[V_{i-1}(x)-\epsilon_{i}\right], \\
& V_{i}(x)=V_{i-1}(x)-\alpha_{i}^{\prime}\left(x, \epsilon_{i}\right) .
\end{aligned}
$$

Suppose now that $V_{i-1}(x)$ is known; then $V_{i}(x)$ becomes determined (see equation (12)) if the solution $\alpha_{i}\left(x, \epsilon_{i}\right)$ of the $i$-th Riccati equation (11) associated to $\epsilon_{i}$ can be found. The key point in this treatment is to realize that there is a simple finite difference formula allowing to find algebraically $\alpha_{i}\left(x, \epsilon_{i}\right)$ in terms of two solutions of the $(i-1)$-th Riccati equation, associated to the factorization energies $\epsilon_{i-1}, \epsilon_{i}[36,52]$ :

$$
\alpha_{i}\left(x, \epsilon_{i}\right)=-\alpha_{i-1}\left(x, \epsilon_{i-1}\right)-\frac{2\left(\epsilon_{i-1}-\epsilon_{i}\right)}{\alpha_{i-1}\left(x, \epsilon_{i-1}\right)-\alpha_{i-1}\left(x, \epsilon_{i}\right)} .
$$

By iterating down this equation it turns out that, at the end, $\alpha_{i}\left(x, \epsilon_{i}\right)$ can be expressed in terms of the $i$ solutions

$$
\alpha_{1}^{\prime}\left(x, \epsilon_{j}\right)+\alpha_{1}^{2}\left(x, \epsilon_{j}\right)=2\left[V_{0}(x)-\epsilon_{j}\right], \quad j=1, \ldots, i,
$$

of the initial Riccati equation, or in terms of the corresponding solutions of the Schrödinger equation,

$$
-\frac{1}{2} u_{j}^{\prime \prime}+V_{0}(x) u_{j}=\epsilon_{j} u_{j}, \quad j=1, \ldots, i,
$$

where $\alpha_{1}\left(x, \epsilon_{j}\right)=u_{j}^{\prime} / u_{j}$.

In order to connect the previous technique and supersymmetric quantum mechanics, let us realize now the standard SUSY algebra with two generators

$$
\left[\mathrm{Q}_{i}, \mathrm{H}_{\mathrm{ss}}\right]=0, \quad\left\{\mathrm{Q}_{i}, \mathrm{Q}_{j}\right\}=\delta_{i j} \mathrm{H}_{\mathrm{ss}}, \quad i, j=1,2,
$$

in the following way $[8,7,11,20,22,31,32,34,43,55]$

$$
\begin{array}{ll}
\mathrm{Q}=\left(\begin{array}{cc}
0 & B_{k}^{+} \\
0 & 0
\end{array}\right), & \mathrm{Q}^{+}=\left(\begin{array}{cc}
0 & 0 \\
B_{k} & 0
\end{array}\right), \\
\mathrm{Q}_{1}=\frac{\mathrm{Q}^{+}+\mathrm{Q}}{\sqrt{2}}, & \mathrm{Q}_{2}=\frac{\mathrm{Q}^{+}-\mathrm{Q}}{i \sqrt{2}}, \quad \mathrm{H}_{\mathrm{ss}}=\left(\begin{array}{cc}
B_{k}^{+} B_{k} & 0 \\
0 & B_{k} B_{k}^{+}
\end{array}\right),
\end{array}
$$

where

$$
B_{k}^{+} B_{k}=\left(H_{k}-\epsilon_{1}\right) \cdots\left(H_{k}-\epsilon_{k}\right), \quad B_{k} B_{k}^{+}=\left(H_{0}-\epsilon_{1}\right) \cdots\left(H_{0}-\epsilon_{k}\right),
$$


$H_{0}$ and $H_{k}$ being the initial and final Hamiltonians, intertwined by $k$-th order differential intertwining operators, namely,

$$
H_{k} B_{k}^{+}=B_{k}^{+} H_{0}, \quad H_{0} B_{k}=B_{k} H_{k}, \quad B_{k}^{+}=A_{k}^{+} \cdots A_{1}^{+}, \quad B_{k}=A_{1}^{-} \cdots A_{k}^{-} .
$$

The initial and final potentials $V_{0}, V_{k}$, are interrelated by:

$$
V_{k}(x)=V_{0}(x)-\sum_{i=1}^{k} \alpha_{i}^{\prime}\left(x, \epsilon_{i}\right)=V_{0}(x)-\left\{\ln \left[W\left(u_{1}, \ldots, u_{k}\right)\right]\right\}^{\prime \prime},
$$

where $W\left(u_{1}, \ldots, u_{k}\right)$ is the Wronskian of the $k$ Schrödinger seed solutions $u_{1}, \ldots, u_{k}$. Let us note a certain resemblance of the $k$-th order SUSY QM presented here with the method of fractional supersymmetry discussed elsewhere [24].

The previous technique has been employed successfully to generate new solvable potentials $V_{k}(x)$ departing from a given initial one $V_{0}(x)$ for several interesting physical systems $[10,21,31,55]$. Of our particular interest is the case of the harmonic oscillator $[13,17,34,35$, $36,38]$, which is worth of an explicit discussion.

\section{$5 \quad$ Harmonic oscillator SUSY partners}

In order to implement the SUSY technique, we need to find first the general solution of the stationary Schrödinger equation (13) for $V_{0}(x)=x^{2} / 2$ and an arbitrary $\epsilon$, which turns out to be:

$$
u=e^{-\frac{x^{2}}{2}}\left[{ }_{1} F_{1}\left(\frac{1-2 \epsilon}{4}, \frac{1}{2} ; x^{2}\right)+2 x \nu \frac{\Gamma\left(\frac{3-2 \epsilon}{4}\right)}{\Gamma\left(\frac{1-2 \epsilon}{4}\right)}{ }_{1} F_{1}\left(\frac{3-2 \epsilon}{4}, \frac{3}{2} ; x^{2}\right)\right],
$$

where ${ }_{1} F_{1}(a, b ; y)$ is the (Kummer) confluent hypergeometric function. Let us perform now a non-singular $k$-th order SUSY transformation which creates precisely $k$ new levels, by simplicity placed below the ground state energy $E_{0}=1 / 2$ of the oscillator [35]. If the factorization energies are ordered as $\epsilon_{k}<\epsilon_{k-1}<\cdots<\epsilon_{1}<1 / 2$, then the non-singular SUSY transformations arise for $\left|\nu_{1}\right|<1,\left|\nu_{2}\right|>1,\left|\nu_{3}\right|<1, \ldots$ The spectrum of the corresponding Hamiltonian reads:

$$
\operatorname{Sp}\left(H_{k}\right)=\left\{\epsilon_{k}, \ldots, \epsilon_{1}, E_{n}=n+1 / 2, n=0,1, \ldots\right\} .
$$

An illustration of a second-order SUSY partner potential of the oscillator for $\left(\epsilon_{1}, \epsilon_{2}\right)=\left(-1,-\frac{6}{5}\right)$ and $\left(\nu_{1}, \nu_{2}\right)=(0,2)$ is shown in Fig. 4 .

Let us note that, for the Hamiltonian $H_{k}$, there exists a natural pair of ladder operators:

$$
L_{k}^{-}=B_{k}^{+} a B_{k}, \quad L_{k}^{+}=B_{k}^{+} a^{+} B_{k}
$$

which are differential operators of order $(2 k+1)$-th satisfying $[17,35,49]$ :

$$
\left[H_{k}, L_{k}^{ \pm}\right]= \pm L_{k}^{ \pm}
$$

By making use of the intertwining relationships (15) and the factorizations of equations (14), it is straightforward to show that:

$$
Q_{2 k+1}\left(H_{k}\right)=L_{k}^{+} L_{k}^{-}=\left(H_{k}-\frac{1}{2}\right) \prod_{i=1}^{k}\left(H_{k}-\epsilon_{i}-1\right)\left(H_{k}-\epsilon_{i}\right) .
$$




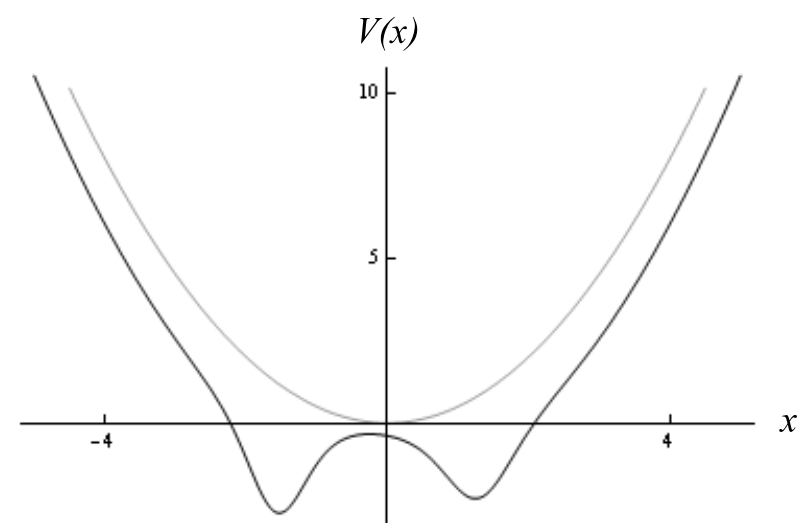

Figure 4. Second-order SUSY partner (black curve) of the oscillator potential (gray curve) generated by taking two Schrödinger solutions of the form given in equation $(16)$ with $\left(\epsilon_{1}, \epsilon_{2}\right)=\left(-1,-\frac{6}{5}\right)$ and $\left(\nu_{1}, \nu_{2}\right)=(0,2)$.

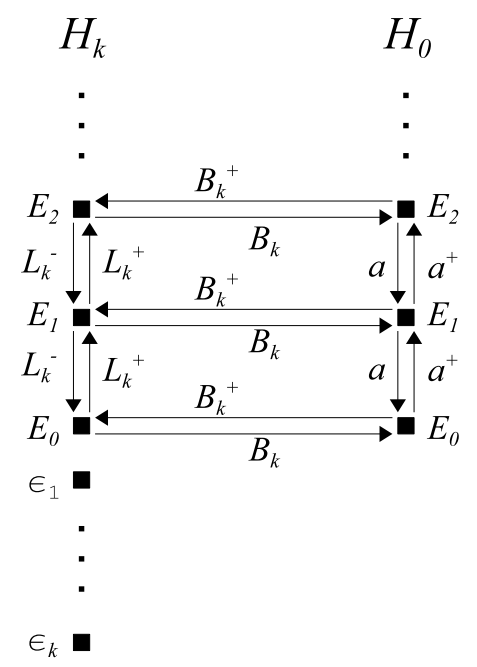

Figure 5. Diagram representing the spectrum and the action of the ladder operators $L_{k}^{ \pm}$for the SUSY partner $H_{k}$ of the harmonic oscillator Hamiltonian $H_{0}$. They are built up from the intertwining operators $B_{k}, B_{k}^{+}$and the harmonic oscillator annihilation and creation operators $a, a^{+}$(see equation (18)).

This implies that the operators $\left\{H_{k}, L_{k}^{-}, L_{k}^{+}\right\}$generate a polynomial Heisenberg algebra of order $(2 k)$-th, which is characterized by equation (19) and the deformed commutator:

$$
\left[L_{k}^{-}, L_{k}^{+}\right]=P_{2 k}\left(H_{k}\right) \text {. }
$$

From the analysis of the roots involved in $Q_{2 k+1}\left(H_{k}\right)$, it turns out that the $\operatorname{Sp}\left(H_{k}\right)$ given in equation (17) can be seen as containing $k+1$ ladders: $k$ of them are one-step ladders, starting and ending at $\epsilon_{j}, j=1, \ldots, k$; in addition, there is an infinite one starting from $\frac{1}{2}[17,35]$. A representation of $\operatorname{Sp}\left(H_{k}\right)$ and the actions of the ladder operators $L_{k}^{ \pm}$are given in Fig. 5 .

We have already all the elements for answering the main question we would like to pose in this paper: since the harmonic oscillator Hamiltonian has an evolution loop, it is natural to ask if its SUSY partners show as well such a kind of closed dynamical processes. First of all let us write down the evolution operator $U(t)=e^{-i H_{k} t}$ associated to $H_{k}$ :

$$
U(t)=\sum_{j=1}^{k} e^{-i \epsilon_{j} t}\left|\psi_{\epsilon_{j}}^{k}\right\rangle\left\langle\psi_{\epsilon_{j}}^{k}\left|+\sum_{n=0}^{\infty} e^{-i\left(n+\frac{1}{2}\right) t}\right| \psi_{n}^{k}\right\rangle\left\langle\psi_{n}^{k}\right| .
$$


If the $k$ factorization energies $\epsilon_{j}, j=1, \ldots, k$ are arbitrary, it turns out that a partial evolution loop is produced for $\tau=2 \pi$ since:

$$
U(2 \pi)=\sum_{j=1}^{k} e^{-i 2 \pi \epsilon_{j}}\left|\psi_{\epsilon_{j}}^{k}\right\rangle\left\langle\psi_{\epsilon_{j}}^{k}\left|+e^{-i \pi} \sum_{n=0}^{\infty}\right| \psi_{n}^{k}\right\rangle\left\langle\psi_{n}^{k}\right| .
$$

This means that any state belonging to the subspace generated by $\left\{\left|\psi_{n}^{k}\right\rangle, n=0,1, \ldots\right\}$,

$$
|\psi(0)\rangle=\sum_{n=0}^{\infty} c_{n}\left|\psi_{n}^{k}\right\rangle, \quad \sum_{n=0}^{\infty}\left|c_{n}\right|^{2}=1,
$$

becomes cyclic with period $\tau=2 \pi$ :

$$
|\psi(\tau)\rangle=e^{-i \pi}|\psi(0)\rangle
$$

A straightforward calculation leads now to the associated geometric phase (see equation (3)):

$$
\beta=-\pi+2 \pi\left\langle\psi(0)\left|H_{k}\right| \psi(0)\right\rangle=2 \pi \sum_{n=1}^{\infty} n\left|c_{n}\right|^{2}
$$

In particular, if $c_{n}=\delta_{n, m}$ it turns out that

$$
\beta=0[\bmod (2 \pi)]
$$

It is interesting as well to evaluate the geometric phases associated to the coherent states which are eigenstates of the annihilation operator of equation (18) [35], namely,

$$
L_{k}^{-}|z\rangle=z|z\rangle, \quad z \in \mathbb{C} .
$$

By expressing $|z\rangle$ in terms of the eigenstates of $H_{k}$,

$$
|z\rangle=\sum_{j=1}^{k} b_{j}\left|\psi_{\epsilon_{j}}^{k}\right\rangle+\sum_{n=0}^{\infty} c_{n}\left|\psi_{n}^{k}\right\rangle
$$

it turns out that

$$
b_{j}=0, \quad c_{n}=\frac{z}{\sqrt{n \prod_{i=1}^{k}\left(n-\epsilon_{i}-\frac{1}{2}\right)\left(n-\epsilon_{i}+\frac{1}{2}\right)}} c_{n-1} .
$$

By iterating down the recurrence relationship for $c_{n}$, it turns out that it becomes expressed in terms of $c_{0}$. The last coefficient is fixed from the normalization condition, leading to:

$$
\begin{aligned}
& |z\rangle=N(r) \sum_{n=0}^{\infty} \frac{z^{n}\left|\psi_{n}^{k}\right\rangle}{\sqrt{n ! \prod_{i=1}^{k} \Gamma\left(n+\frac{1}{2}-\epsilon_{i}\right) \Gamma\left(n+\frac{3}{2}-\epsilon_{i}\right)}}, \\
& N(r)=\sqrt{\frac{\prod_{i=1}^{k} \Gamma\left(\frac{1}{2}-\epsilon_{i}\right) \Gamma\left(\frac{3}{2}-\epsilon_{i}\right)}{{ }_{0} F_{2 k}\left(\frac{1}{2}-\epsilon_{1}, \ldots, \frac{1}{2}-\epsilon_{k}, \frac{3}{2}-\epsilon_{1}, \ldots, \frac{3}{2}-\epsilon_{k} ; r^{2}\right)}},
\end{aligned}
$$




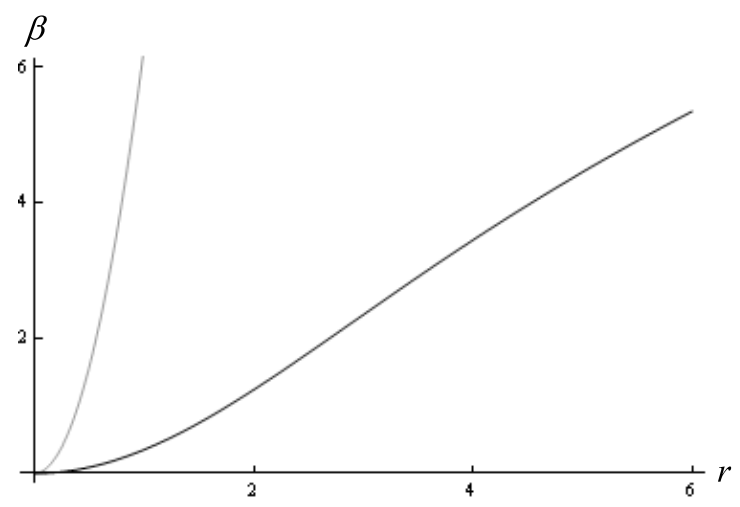

Figure 6. Plot of the geometric phase $\beta$ of equation (22) as a function of $r$ (black curve). The geometric phase associated to the standard coherent states $\beta=2 \pi r^{2}$ is as well plotted (gray curve).

where $r=|z|$. By using equation (20), the associated geometric phase becomes now:

$$
\beta=\frac{2 \pi r^{2}}{\prod_{i=1}^{k}\left(\frac{1}{2}-\epsilon_{i}\right)\left(\frac{3}{2}-\epsilon_{i}\right)} \frac{{ }_{0} F_{2 k}\left(\frac{3}{2}-\epsilon_{1}, \ldots, \frac{3}{2}-\epsilon_{k}, \frac{5}{2}-\epsilon_{1}, \ldots, \frac{5}{2}-\epsilon_{k} ; r^{2}\right)}{{ }_{F_{2 k}\left(\frac{1}{2}-\epsilon_{1}, \ldots, \frac{1}{2}-\epsilon_{k}, \frac{3}{2}-\epsilon_{1}, \ldots, \frac{3}{2}-\epsilon_{k} ; r^{2}\right)} .}
$$

Let us note that, for $k=1$ and $\epsilon_{1}=-1 / 2$, the expression of equation $(21)$ reduces $(\bmod (2 \pi))$ to the expression of equation (27) of [30] since

$$
\beta_{G C S}-2 \pi=2 \pi\left[\frac{{ }_{0} F_{2}\left(1,1 ; r^{2}\right)}{{ }_{0} F_{2}\left(1,2 ; r^{2}\right)}-1\right]=\pi r^{2} \frac{{ }_{0} F_{2}\left(2,3 ; r^{2}\right)}{{ }_{0} F_{2}\left(1,2 ; r^{2}\right)} .
$$

On the other hand, for $\left(\epsilon_{1}, \epsilon_{2}\right)=\left(-1,-\frac{6}{5}\right)$ it turns out that

$$
\beta=\frac{160 \pi r^{2}{ }_{0} F_{4}\left(\frac{5}{2}, \frac{7}{2}, \frac{27}{10}, \frac{37}{10} ; r^{2}\right)}{1377} \frac{{ }_{0} F_{4}\left(\frac{3}{2}, \frac{5}{2}, \frac{17}{10}, \frac{27}{10} ; r^{2}\right)}{\text {. }}
$$

A plot of this geometric phase as a function of $r$ is shown in Fig. 6 (black curve). The geometric phase acquired by the standard coherent states, which turns out to be $\beta=2 \pi r^{2}$, is as well drawn (gray curve).

Coming back to our general subject, if the factorization energies are such that

$$
\epsilon_{j}=\frac{1}{2}-\frac{l_{j}}{m_{j}} \quad \forall j=1, \ldots, k,
$$

where $l_{j}, m_{j}$ are coprime, then a global evolution loop of period $\tau=2 M \pi$ is obtained, namely,

$$
\begin{aligned}
U(2 M \pi) & =e^{-i M \pi}\left(\sum_{j=1}^{k} e^{i 2 \pi l_{j} \frac{M}{m_{j}}}\left|\psi_{\epsilon_{j}}^{k}\right\rangle\left\langle\psi_{\epsilon_{j}}^{k}\left|+\sum_{n=0}^{\infty} e^{-i 2 \pi M n}\right| \psi_{n}^{k}\right\rangle\left\langle\psi_{n}^{k}\right|\right) \\
& =e^{-i M \pi}\left(\sum_{j=1}^{k}\left|\psi_{\epsilon_{j}}^{k}\right\rangle\left\langle\psi_{\epsilon_{j}}^{k}\left|+\sum_{n=0}^{\infty}\right| \psi_{n}^{k}\right\rangle\left\langle\psi_{n}^{k}\right|\right)=e^{-i M \pi} I,
\end{aligned}
$$

with $M$ being the least common multiple of $\left\{m_{j}, j=1, \ldots, k\right\}$. In this case any arbitrary initial state

$$
|\psi(0)\rangle=\sum_{j=1}^{k} b_{j}\left|\psi_{\epsilon_{j}}^{k}\right\rangle+\sum_{n=0}^{\infty} c_{n}\left|\psi_{n}^{k}\right\rangle, \quad \sum_{j=1}^{k}\left|b_{j}\right|^{2}+\sum_{n=0}^{\infty}\left|c_{n}\right|^{2}=1,
$$


is cyclic with period $\tau=2 M \pi$ :

$$
|\psi(\tau)\rangle=e^{-i M \pi}|\psi(0)\rangle .
$$

The associated geometric phase becomes finally:

$$
\beta=-M \pi+2 M \pi\left(\sum_{j=1}^{k} \epsilon_{j}\left|b_{j}\right|^{2}+\sum_{n=0}^{\infty}\left(n+\frac{1}{2}\right)\left|c_{n}\right|^{2}\right)=2 M \pi\left(\sum_{n=1}^{\infty} n\left|c_{n}\right|^{2}-\sum_{j=1}^{k} \frac{l_{j}}{m_{j}}\left|b_{j}\right|^{2}\right) .
$$

\section{Conclusions}

In this paper it has been shown that the SUSY partners of the harmonic oscillator Hamiltonian realize straightforwardly the polynomial Heisenberg algebras of order $2 k$. As a consequence, if the SUSY transformation creates $k$ new levels for $H_{k}$, the corresponding spectrum can be seen as composed of $k+1$ independent ladders, $k$ of them being one-step ladders and an infinite one starting from $E_{0}=1 / 2$. It has been proven also that the corresponding Hamiltonians present in general the so-called partial evolution loops, which induce cyclic evolutions in the subspace generated by the eigenstates associated to $E_{n}$ and, consequently, have associated geometric phases. This applies, in particular, to the coherent states which are eigenstates of the natural annihilation operator of the system, for which a general formula for the associated geometric phase has been derived in this paper. In particular, from this general result we have recovered the expression for the geometric phase which was found previously [30] for the Abraham-Moses family of potentials isospectral to the harmonic oscillator. Finally, we have shown that it is possible to produce global evolution loops by imposing restrictions on the involved factorization energies. The associated geometric phases induced by the last operator identity have been as well evaluated.

\section{Acknowledgements}

The author acknowledges the financial support of Conacyt, project 152574, as well as the comments of Alonso Contreras-Astorga.

\section{References}

[1] Adler V.E., Nonlinear chains and Painlevé equations, Phys. D 73 (1994), 335-351.

[2] Aharonov Y., Anandan J., Phase change during a cyclic quantum evolution, Phys. Rev. Lett. 58 (1987), $1593-1596$.

[3] Aizawa N., Sato H.T., Isospectral Hamiltonians and $W_{1+\infty}$ algebra, Progr. Theoret. Phys. 98 (1997), 707718, quant-ph/9601026.

[4] Anandan J., Christian J., Wanelik K., Resource letter GPP-1: geometric phases in physics, Amer. J. Phys. 65 (1997), 180-185, quant-ph/9702011.

[5] Andrianov A.A., Cannata F., Nonlinear supersymmetry for spectral design in quantum mechanics, J. Phys. A: Math. Gen. 37 (2004), 10297-10321, hep-th/0407077.

[6] Andrianov A.A., Cannata F., Ioffe M.V., Nishnianidze D.N., Systems with higher-order shape invariance: spectral and algebraic properties, Phys. Lett. A 266 (2000), 341-349, quant-ph/9902057.

[7] Andrianov A.A., Ioffe M.V., Cannata F., Dedonder J.P., Second order derivative supersymmetry, $q$-deformations and the scattering problem, Internat. J. Modern Phys. A 10 (1995), 2683-2702, hep-th/9404061.

[8] Andrianov A.A., Ioffe M.V., Spiridonov V.P., Higher-derivative supersymmetry and the Witten index, Phys. Lett. A 174 (1993), 273-279, hep-th/9303005. 
[9] Arık M., Atakishiyev N.M., Wolf K.B., Quantum algebraic structures compatible with the harmonic oscillator Newton equation, J. Phys. A: Math. Gen. 32 (1999), L371-L376.

[10] Bagchi B.K., Supersymmetry in quantum and classical mechanics, Chapman \& Hall/CRC Monographs and Surveys in Pure and Applied Mathematics, Vol. 116, Chapman \& Hall/CRC, Boca Raton, FL, 2001.

[11] Bagrov V.G., Samsonov B.F., Darboux transformation of the Schrödinger equation, Phys. Part. Nuclei 28 (1997), 374-397.

[12] Baye D., Sparenberg J.M., Inverse scattering with supersymmetric quantum mechanics, J. Phys. A: Math. Gen. 37 (2004), 10223-10249.

[13] Bermúdez D., Fernández D.J., Non-Hermitian Hamiltonians and the Painlevé IV equation with real parameters, Phys. Lett. A 375 (2011), 2974-2978, arXiv:1104.3599.

[14] Bermúdez D., Fernández D.J., Supersymmetric quantum mechanics and Painlevé IV equation, SIGMA 7 (2011), 025, 14 pages, arXiv:1012.0290.

[15] Berry M.V., Quantal phase factors accompanying adiabatic changes, Proc. Roy. Soc. London Ser. A 392 (1984), 45-57.

[16] Bohm A., Boya L.J., Kendrick B., Derivation of the geometrical phase, Phys. Rev. A 43 (1991), 1206-1210.

[17] Carballo J.M., Fernández D.J., Negro J., Nieto L.M., Polynomial Heisenberg algebras, J. Phys. A: Math. Gen. 37 (2004), 10349-10362.

[18] Cariñena J.F., Perelomov A.M., Rañada M.F., Isochronous classical systems and quantum systems with equally spaced spectra, J. Phys. Conf. Ser. 87 (2007), 012007, 14 pages.

[19] Chruściński D., Jamiołkowski A., Geometric phases in classical and quantum mechanics, Progress in Mathematical Physics, Vol. 36, Birkhäuser Boston Inc., Boston, MA, 2004.

[20] Contreras-Astorga A., Fernández D.J., Supersymmetric partners of the trigonometric Pöschl-Teller potentials, J. Phys. A: Math. Theor. 41 (2008), 475303, 18 pages, arXiv:0809.2760.

[21] Cooper F., Khare A., Sukhatme U., Supersymmetry in quantum mechanics, World Scientific Publishing Co. Inc., River Edge, NJ, 2001.

[22] Correa F., Jakubský V., Nieto L.M., Plyushchay M.S., Self-isospectrality, special supersymmetry, and their effect on the band structure, Phys. Rev. Lett. 101 (2008), 030403, 4 pages, arXiv:0801.1671.

[23] Daoud M., Kibler M.R., Bosonic and $k$-fermionic coherent states for a class of polynomial Weyl-Heisenberg algebras, J. Phys. A: Math. Theor. 45 (2012), 244036, 22 pages, arXiv:1110.4799.

[24] Daoud M., Kibler M.R., Fractional supersymmetry and hierarchy of shape invariant potentials, J. Math. Phys. 47 (2006), 122108, 11 pages, quant-ph/0609017.

[25] Daoud M., Kibler M.R., Phase operators, temporally stable phase states, mutually unbiased bases and exactly solvable quantum systems, J. Phys. A: Math. Theor. 43 (2010), 115303, 18 pages, arXiv:1002.0955.

[26] Dong S.H., Factorization method in quantum mechanics, Fundamental Theories of Physics, Vol. 150, Springer, Dordrecht, 2007.

[27] Dubov S.Y., Eleonsky V.M., Kulagin N.E., Equidistant spectra of anharmonic oscillators, Sov. Phys. JETP 75 (1992), 446-451.

[28] Emmanouilidou A., Zhao X.G., Ao P., Niu Q., Steering an eigenstate to a destination, Phys. Rev. Lett. 85 (2000), 1626-1629.

[29] Fernández D.J., Bogdan Mielnik: contributions to quantum control, in Geometric Methods in Physics (XXX Workshop, Bialowieza, Poland, June 26 - July 2, 2011), Editors P. Kielanowski, S.T. Ali, A. Odzijewicz, M. Schlichenmaier, T. Voronov, to appear.

[30] Fernández D.J., Geometric phases and Mielnik's evolution loops, Int. J. Theor. Phys. 33 (1994), 2037-2047, hep-th/9410213.

[31] Fernández D.J., Supersymmetric quantum mechanics, AIP Conf. Proc. 1287 (2010), 3-36, arXiv:0910.0192.

[32] Fernández D.J., SUSUSY quantum mechanics, Internat. J. Modern Phys. A 12 (1997), 171-176, quant-ph/9609009.

[33] Fernández D.J., Transformations of a wave packet in a Penning trap, Nuovo Cimento B 107 (1992), 885-893.

[34] Fernández D.J., Fernández-García N., Higher-order supersymmetric quantum mechanics, AIP Conf. Proc. 744 (2005), 236-273, quant-ph/0502098.

[35] Fernández D.J., Hussin V., Higher-order SUSY, linearized nonlinear Heisenberg algebras and coherent states, J. Phys. A: Math. Gen. 32 (1999), 3603-3619. 
[36] Fernández D.J., Hussin V., Mielnik B., A simple generation of exactly solvable anharmonic oscillators, Phys. Lett. A 244 (1998), 309-316.

[37] Fernández D.J., Mielnik B., Controlling quantum motion, J. Math. Phys. 35 (1994), 2083-2104.

[38] Fernández D.J., Negro J., Nieto L.M., Elementary systems with partial finite ladder spectra, Phys. Lett. A 324 (2004), 139-144.

[39] Fernández D.J., Nieto L.M., del Olmo M.A., Santander M., Aharonov-Anandan geometric phase for spin- $\frac{1}{2}$ periodic Hamiltonians, J. Phys. A: Math. Gen. 25 (1992), 5151-5163.

[40] Fernández D.J., Rosas-Ortiz O., Inverse techniques and evolution of spin-1/2 systems, Phys. Lett. A 236 (1997), 275-279.

[41] Gangopadhyaya A., Mallow J.V., Rasinariu C., Supersymmetric quantum mechanics, World Scientific, Singapore, 2011.

[42] Horváthy P.A., Plyushchay M.S., Valenzuela M., Bosons, fermions and anyons in the plane, and supersymmetry, Ann. Physics 325 (2010), 1931-1975, arXiv:1001.0274.

[43] Ioffe M.V., Nishnianidze D.N., SUSY intertwining relations of third order in derivatives, Phys. Lett. A 327 (2004), 425-432, hep-th/0404078.

[44] Lin Q.G., Time evolution, cyclic solutions and geometric phases for general spin in an arbitrarily varying magnetic field, J. Phys. A: Math. Gen. 36 (2003), 6799-6806, quant-ph/0307099.

[45] Lin Q.G., Time evolution, cyclic solutions and geometric phases for the generalized time-dependent harmonic oscillator, J. Phys. A: Math. Gen. 37 (2004), 1345-1371, quant-ph/0402159.

[46] Marquette I., Superintegrability and higher order polynomial algebras, J. Phys. A: Math. Theor. 43 (2010), 135203, 15 pages, arXiv:0908.4399.

[47] Mateo J., Negro J., Third-order differential ladder operators and supersymmetric quantum mechanics, J. Phys. A: Math. Theor. 41 (2008), 045204, 28 pages.

[48] Mielnik B., Evolution loops, J. Math. Phys. 27 (1986), 2290-2306.

[49] Mielnik B., Factorization method and new potentials with the oscillator spectrum, J. Math. Phys. 25 (1984), $3387-3389$.

[50] Mielnik B., Global mobility of Schrödinger's particle, Rep. Math. Phys. 12 (1977), 331-339.

[51] Mielnik B., Space echo, Lett. Math. Phys. 12 (1986), 49-56.

[52] Mielnik B., Nieto L.M., Rosas-Ortiz O., The finite difference algorithm for higher order supersymmetry, Phys. Lett. A 269 (2000), 70-78, quant-ph/0004024.

[53] Mielnik B., Ramírez A., Ion traps: some semiclassical observations, Phys. Scr. 82 (2010), 055002, 13 pages.

[54] Mielnik B., Ramírez A., Magnetic operations: a little fuzzy mechanics?, Phys. Scr. 84 (2011), 045008, 17 pages, arXiv:1006.1944.

[55] Mielnik B., Rosas-Ortiz O., Factorization: little or great algorithm?, J. Phys. A: Math. Gen. 37 (2004), 10007-10035.

[56] Mostafazadeh A., Dinamical invariants, adiabatic approximation and the geometric phase, Nova Science Publishers Inc., New York, 1992.

[57] Plyushchay M.S., Deformed Heisenberg algebra with reflection, Nuclear Phys. B 491 (1997), 619-634, hep-th/9701091.

[58] Shapere A., Wilczek F. (Editors), Geometric phases in physics, Advanced Series in Mathematical Physics, Vol. 5, World Scientific Publishing Co. Inc., Teaneck, NJ, 1989.

[59] Sukumar C.V., Supersymmetric quantum mechanics and its applications, AIP Conf. Proc. 744 (2005), 166-235.

[60] Veselov A.P., Shabat A.B., Dressing chains and the spectral theory of the Schrödinger operator, Func. Anal. Appl. 27 (1993), 81-96. 